CIENCIA Y SOCIEDAD

Volumen $V$, Número 2

Julio-Diciem bre 1981

LA EXISTENCIA DE UN

MERCADO POLITICO EN EL

PROCESO DE LA POLITICA

PUBLICA: EI caso de la política de

precios del arroz en la República

Dominicana

MIGUEL SANG BEN

CAPITULO I

FUNDAMENTOS CONCEPTUALES

\title{
I. Antecedentes:
}

La formulación de la política económica en los países subdesarrollados es un proceso muy complejo. Cada institución puede producir y adquirir metas y funciones divergentes, diferentes de las que originalmente fueron estipuladas en la ley o decreto que la estableció. Estos cambios son causados principalmente por presiones políticas, pero no hay documentación sólida sobre este conflic to y mucho menos sobre las instituciones tercermundistas.

Este es el caso del Instituto de Estabilización de Precios, de la República Dominicana (que llamaremos "el Instituto" en el resto de este trabajo) en particular, así como el de los países latino-americanos con instituciones creadas con el propósito de canalizar y administrar el Programa de Alimentos para la Paz de los

Basado en la investigación de grado para optar al Master in Arts in Social Sciences presentada a la Universidad de Chicago. 
E.U. y los embarques P.L. 480. (La tabla 1 muestra la importancia del comercio alimenticio internacional para América Latina.)

Algunos ejemplos de países latino-americanos son: el Instituto de Comercilización Agrícola de Guatemala, la Corporación de Mercadeo Agrícola de Venezuela, la Empresa Nacional de Almacenamiento y Comercialización de Ecuador, el Instituto de Mercadeo Agrícola de Colombia, el Instituto Hondureño de Mercadeo Agrícola y la Empresa Cubana Importadora de Alimentos.

La experiencia del autor en el Instituto Dominicano es la principal fuente de información de este ensayo. Para lograr sus variados objetivos económicos, estas instituciones han desarrollado una gama completa de técnicas de intervención económica. ${ }^{1}$

1. Técnicas de Subsidios. El Instituto tiene el poder de elegir un variado programa de mercadeo direc to de productos alimenticios y factores de producción agrícola. En el pasado, éstas eran organizadas esporádicamente; sin embargo, un programa definitivo fue establecido durante el período de emergencia que siguió a la devastación del país por los huracanes David y Federico en el 1979. El Instituto tiene que financiar estos subsidios de las ganancias obtenidas de los mercados monopolizados de que disfruta.

2. Técnicas de Regulación: Los principales artículos de primera necesidad de la dieta diaria son monopolizados por el Institu to. El arroz es comprado por los molinos, pero el producto final debe ser vendido al Instituto, y él, entonces, lo vende a los mayoristas y a los detallistas. Otro artículo regulado es el aceite vegetal. Hay un déficit crónico de aceite vegetal en el país, pero el Instituto es el único importador. Esta disposición produce un diferencial de precio en favor del Instituto que le permite subsidiar otros programas. Hay también un arreglo similar para el maíz.

3. Técnicas de Manipulación: Como monopolista de algunos de los artículos más importantes en la dieta dominicana, el Instituto usa su poder administrativo para intervenir en el mercado. El procedimiento más importante es el sistema de "cuota", usado para asignar una cantidad, de cada artículo que mercadea el Instituto a cada comerciante, de acuerdo al valor de la patente.

Estos tres conjuntos de técnicas de intervención, coexistentes en algunos de los mercados más importantes de alimentos en la República Dominicana, son la causa de algunas de las más considerables deformaciones en la econom ía:

1. A nivel de la Producción: La mitad del número total de los molinos

1. Estas categorías son de:

R.Ripley, ed. Public Policies and their Politics. New York, N.Y., Norton, 1966. 
arroceros están cerrados actualmente. La producción de arroz ha tenido altas y sentadas más adelante.
sajas en leformaciones producidas a este nivel serán pre-

2. A Nivel del Consumo: Las ventas de productos alimenticios del Instituto son hechas con una política de sólo dinero en efectivo debido a los problemas administrativos en las cuentas a crédito. Esto produce otra deformación en los precios de consumo: sólo los grandes comerciantes pueden sobrevivir a la política de ventas en efectivo y ellos imponen arreglos financieros arbitrarios a los pequeños comerciantes. El precio del producto final es deformado.

3. En el Nivel de Balanza de Pagos: El estancamiento virtual de la producción agrícola y las limitaciones de almacenamiento del Instituto han requerido un programa creciente de importaciones alimenticias que presionan la balanza de pagos.

Esta situación de múltiples deformaciones puede sér explicada en forma limitada por técnicas económicas que dejan sin contestar por qué estas disposiciones se mantienen.

Un enfoque es el proveniente de la teoría económica aplicada a los fenómenos políticos, ejemplificado por el artículo del Profesor Theodore Schultz, ${ }^{2}$ donde explica la existencia de un "mercado político" en la toma de decisiones de precios agrícolas.

El artículo del Profesor Schultz empieza con el análisis de los países que:

1.- No sobre-valúan o sub-valúan la producción agrícola, que serían los estados ideales de no deformación en los mercados agrícolas.

2.- La producción agrícola sobrevaluada, principalmente en países desarrollados con al tos ingresos qué les permiten sostener esos precios.

3. La producción agrícola sub-valuada, principalmente en países sub-desarrollados incapaces de aumentar su producción agrícola.

La economía de sobre y sub-evaluación de la producción, puede ser presentada en estas frases: Cuando la producción es sobrevaluada, los incentivos a los

2. T.W. Schultz. "On Economics and Politics of Agricultre". En su : Distortions of Agricultural Incentives. Bloomington, Ind, Indiana University Press, 1978.

Un enfoque más formal y general se encuentra en:

G. Becker. "Competition and Democracy". In : The Economics Approach to Human Behavior. Chicago, III, University of Chicago Press, 1976. 
productores son más altos que el óptimo; cuando es sub-valuada, los incentivos a la producción están por debajo del nivel óptimo. El Profesor Schultz desarrolla sus argumentos basándose en los efectos de la sub-valuación en países de bajas entradas o sub-desarrollados. A continuación presentamos el argumento del Profesor Schultz tomando una larga cita de su artículo:

No es cinismo decir que los gobiernos hagan lo que estimen necesario para sobrevivir. Los individuos que gobiernan son políticamente dependientes del apoyo de grupos de población particulares que hacen el régimen factible. Las políticas económicas son, en este contexto, un medio de mantener apoyo político. En las políticas que son seguidas, no hay falta de racionalizaciones de parte de los encargados del gobierno y de los teóricos que les sirven. Aunque es debatible cuánto aprenden los gobiernos de sus errores en asuntos económicos, ellos aprenden lentamente comparados a la habilidad de los agricultores en aprender de su experiencia. Muy poca atención, sin embargo, ha sido dada a los factores que determinan la función de aprendizaje de los gobiernos.

Como se ha notado ya, hay un "mercado político". Las consecuencias económicas de este mercado es muy diferente de ésas de un mercado competitivo y abierto que no es controlado por el gobierno y no es manipulado privativamente. La teoría económica se extiende para mostrar las implicaciones del mercado político y para probar las implicaciones contra el comportamiento observable. La suposición yacente de esta extensión de la economía es que la función del mercado político es incrementar los beneficios económicos que son pedidos por individuos y grupos de individuos que tienen acceso e influencia en el mercado político.

Hay muchas "razones" por las cuales los gobiernos sub-valúan las contribuciones económicas de la agricultura. Estas razones están encadenadas a varias ideologías, la mayoría de las cuales han sido "importadas" de países de altos ingresos.

Aunque la población rural en los países de bajos ingresos es, cón mucho la mayor, el mercado político favorece fuertemente a la población urbana, a expensas directa de los campesinos. Políticamente, los consumidores urbanos y la industria demandan comida barata... Más aún, la sub-valuación de la agricultura por parte del gobierno es aún racionalizada por un aparato de argumentos que incluye la herencia colonial, el atraso de la agricultura y su presumida baja contribución al desarrollo económico. La agricultura en muchos paises de bajos ingresos lleva el estigma de haber sido el suplidor de la materia prima y de algunos productos alimenticios que sirvieron al interés económico de los poderes coloniales. Todavía es ampliamente sostenida la opinión de que la agricultura es inherentemente un sector atrasado; 
la presunción es que la población rural resiste la modernización a pesar de las pequeñas "revoluciones verdes". Desde el punto de vista de los que administran el mercado político, el pequeño cultivador es visto como indiferente a los incentivos económicos, porque se presume que él está fuertemente atado a su tradicional bajo nivel de productividad. Así, el escenario está listo para la doctrina que postula que la industrialización es la principal base del desarrollo económico y la economía política está designada para dar prioridad tope a la industria y esto incluye granos alimenticios baratos.

Aún otra razón, a menudo citada, del por qué los gobiernos de algunos países de bajos ingresos abandonan la agricultura, relata los presumidos efectos adversos de los precios internacionales y los precios del mercado mundial para productos agrícolas y los gastos (por ejemplo, los fertilizantes) que han sido inestables.

Es verdad que los eventos de los precios mundiales asociados con la Primera Guerra Mundial, la Gran Depresión, la Segunda Guerra Mundial y la reciente inflación mundial compuesta por el monopolio de los precios del petróleo y los efectos de precios de las cosechas cortas, han causado una gran inestabilidad económica. Se habla mucho también de la declinación secular en los términos de la venta de las cosechas agrícolas, pero esta declinación refleja en gran medida el declive relativo en los costos reales de la producción de las cosechas...

Actualmente se avanzan varios argumentos en beneficio de consideraciones de bienestar con la implicación de que la eficiencia en la producción agricola es en muchos puntos inconsistente con el bienestar de la población. Este concepto de bienestar ha sido también "importado" de los países de altos ingresos. Se caracteriza por impuestos sobre la renta progresivos con una substancial parte de la renta coloćada a personas de bajos ingresos como transferencias de ingresos. Lo que estos países de al tos ingresos hacen al cobrar ingresos públicos y al hacer transferencia de ingresos, está muy lejos de la procuración de los granos alimenticios de los granjeros para suplir a las tiendas urbanas de "precio justo" y por este medio subsidiar a los consumidores a expensas de la agricultura. ${ }^{3}$

El análisis del Profesor Schultz nos lleva a concluir que el efecto más peligroso en la producción agrícola de este mercado político es la supresión del papel de los campesinos como empresarios. Esta conclusión requiere el establecimiento de un papel apropiado para la intervención gubernamental en los mercados agrícolas.

3. Schultz. On Economics... p. 10-12. 
En un trabajo previo, el Profesor Schuitz presenta muy adecuadamente este papel gubernamental: Invertir en la formación de capital humano en la agricultura a través de la difusión de nuevas técnicas y reducir el riesgo en la adopción de nuevas tecnologías. ${ }^{4}$ Este papel debe estar presente ep el mercado político si los agentes de las nuevas tecnologías lo requieren del gobierno. La producción de agricultura moderna requiere grandes inversiones públicas, pero éstas no son óptimamente colocadas porque los agentes en el mercado político sufren la misma miopía gubernamental: ellos sólo ven los beneficios a cor to plazo.

\section{Comentarios Finales:}

La hipotesis del Profesor Schultz es una explicación práctica de las deformaciones de la economía política en la producción agrícola, y se ajusta a nuestro caso del Instituto mejor que los enfoques económicos y organizacionales.

Parece ser que la hipótesis del Mercado Político puede ser propiamente tratada en una investigación profunda. Sin embargo, trataremos de establecer el caso del Instituto de Estabilización de Precios usando la hipótesis del Profesor Schultz. Nos parece que está suficientemente claro en esta etapa porque tenemos informaciones adicionales y experiencia como funcionario de ese Instituto. Nos limitaremos al estudio de la política del arroz porque es el artículo más importante y con la historia más larga de intervención estatal. Es el caso más completo de deformación provocada por el monopolio de poder del Instituto en este mercado. Este proceso de intervención estatal refleja una cadena de eventos en el "mercado político" que requiere una intervención gubernamental incrementáda en este mercado.

Es apropiado enfatizar la importancia de este problema en las palabras del Profesor Schultz: "Los incentivos son importantes. Los grandes aumentos potenciales en la producción agrícola en los países de bajos ingresos no pueden ser realizados mientras los empresarios agrícolas confronten los incentivos erróneos". 5

4. T.W. Schultz. Transforming Traditional Agriculture. New Haven, Conn., Yale University Press, 1964.

5. Schultz. On Economics... p. 19. 


\section{LA INTER VENCION DEL GOBIERNO DOMINICANO - EN EL MERCADO DEL ARROZ}

\section{Antecedentes}

El Instituto de Estabilización de Precios está a cargo de la regulación y la estabilización del precio interno de los productos agrícolas y los factores de producción agropecuaria. Actualmente, el Instituto regula el proceso de mercadeo y los precios mínimos de una docena de productos agrícolas importantes. Así mismo, controla el mercadeo legal de todo el arroz y el azúcar.

En los últimos cinco años, se ha permitido una alza muy grande a los preciosinternos. Actualmente los precios internos son más altos que los precios de importación. En el caso del arroz esta diferencia es de 40 por ciento (ver tabla 2).

Estos diferenciales, sin embargo, no son obstáculo para aumentar las impotaciones, principalmente de arroz y aceite vegetal (ver tabla 3).

En este capítulo presentaremos la situación del arroz, un recuento histórico de la formación del plan de acción y una in terpretación del mercado político bajo este proceso.

\section{Producción y Estadísticas de Precios:}

La estadística de la producción de arroz muestra una tendencia a marcadas fluctuaciones (ver tabla 4). Actualmente los cambios porcentuales fluctúan entre 22.08 en $1976-77$ y 27.57 en 1974-75. Durante el período 1960-78, la producción de arroz aumentó 85.9 por ciento en un promedio anual de 4.77 por ciento. Estas fluctuaciones son los principales indicadores para guiar a los burócratas gubernamentales en sus decisiones de acción.

El objetivo explícito ha sido precios estables por un periodo relativamente largo. La tabla 5 muestra que este objetivo ha tenido un patrón muy inestable con una tendencia hacia precios más altos. Sin embargo, para obtener este objetivo a corto plazo, el Instituto ha tenido que establecer un programa de importación masiva. Este proceso es el obje to principal del intercambio de influencia política en la formulación de normas relacionadas a las importaciones y producción de arroz en la República Dominicana.

\section{Modelo de Intervención: ${ }^{6}$}

6. Basado en la colección de leyes y decretos relacionados con INESPRE. 
La institución reguladora más antigua es la Dirección General de Control de Precios, de la Secretaría de Industria y Comercio, establecida en 1963 por la Ley 13. Esta empezó un proceso que culmina con el Instituto de Estabilización de Precios, establecido por la Ley 8 , el día 2 de diciembre de 1969, y con la Ley 133 , el 21 de mayo de 1971, estableciendo el Centro Dominicano de Promoción de Exportaciones. Estas instituciones, junto a la Secretaría de Agricultura y sus dependencias; son los instrumentos de intervención gubernamental en los mercado agrícolas. La influencia del gobierno en los mercados fue desarrollada rápidamente durante los últimos cinco años del régimen del Dr. Joaquín Balaguer (1973-78). Este hecho nos permite comprender la mezcla del estilo político del Dr. Balaguer y del proceso de formación de política en el Instituto.

El "Modelo de Intervención" resultante puede ser presentado como un modelo de sistemas con componentes especializados. Este modelo es el marco básico para entender las diversas oportunidades para influenciar el proceso que tienen personas extrañas al gobierno.

El diagrama No. 1 presenta el "Sistema de Control y Regulación de Productos Agrícolas". Los principales componentes son el Ejecutivo, el Congreso y las Agencias Regulatorias.. Las últimas ejecutan las medidas decididas por el primero.

El flujo específico de orden en este modelo es el siguiente:

1. Medidas de control y regulación son dictadas por el Ejecutivo -el-Presidente - a través de decretos o por leyes que emanan del Congreso.

2. Las Agencias Reguladoras (el Instituto, el Control de Precios, la Secretaría de Agricultura, etc.) ejecutan estas medidas de acuerdo a la naturaleza de las mismas, precio, producción, exportación o importación, etc.

3. Esta acción regulatoria o de control afecta el medio de suministros y demandas de un artículo en particular, modificando el comportamiento de los productores y consumidores.

4. El comportamiento del productor y consumidor produce una reacción, una presión en el gobierno -exclusivamente en el Ejecutivo-para conseguir una política más ventajosa.

La aplicación particular al mercado de arroz es más compleja debido al hecho de que el Estado controla parte de la producción a través de los asentamientos de la Reforma Agraria. El diagrama 2 muestra la particular intervención del mercado de arroz. Esta compleja estructura puede ser explicada por el siguiente recuento histórico. 


\section{Políticas en la Intervención del Mercado Arrocero: Un Recuento Histórico 1969.78}

Esta parte del ensayo tratará de la secuencia de medidas y políticas implementadas por el Gobierno Dominicano, para probar el "mercado político" a este
nivel.

Al final de 1969 , la cosecha de arroz excedió en un 12 por ciento el consumo nacional. Esto significó un exceso de cerca de 50,000 toneladas métricas de arroz en cáscara. Al principio del 1970, el exceso aumentó a casi 100,000 toneladas métricas. Con el sólo propósito de mantener los precios altos en la finca, el Instituto, recientemente creado, fue forzado a comprar, a relativos altos precios, todo el exceso. Esto marca la primera intervención en el mercado arrocero del Instituto. La presión campesina forzó al Presidente a activar algunas leyes liberales de Reforma Agraria. Estas leyes crearon importantes cambios a nivel de la producción de arroz.

La Ley No. 290 declara de interés nacional la transferencia al Instituto Agrario Dominicano de todas las tierras irrigadas por canales de irrigación pública usados en la producción de arroz. El establecimiento de cooperativas agrícolas fue legalizado por la Ley No. 391 en el año de 1972. En este año una escasez de arroz fue prevista. La tabla 4 muestra una virtual declinación en producción debido a que agricultores privados estaban descontentos con las leyes agrarias.

Se esperaba que los precios subieran, asi que tres alternativas fueron presentadas al Instituto y al Ejecutivo:

1. Importar una reserva

2. Declarar un monopolio de arroz en cáscara de parte del Institu to

3. Declarar al Instituto el único comprador de arroz en cáscara.

La primera opción fue elegida por una comisión establecida por el Decreto 2431. Dos meses más tarde, las primeras 2,000 toneladas métricas llegaron al puerto de Santo Domingo. La incompetencia del gobierno para producir arroz en miles de acres dados al Instituto fue un factor principal en el plan de la gran im. portación. Para enfrentar un problema de producción repentino; se recomendó como política mantener por lo menos 20,000 toneladas métricas en reserva. La tabla 6 muestra las grandes importaciones hechas por el Instituto durante 1972 y 1978. El único actor que se benefició de este plan fueron los grandes mayoristas. Algunos mercados negros y mecanismos corruptos fueron desplegados para vender este arroz importado con grandes márgenes de beneficios. Aun con estas importaciones, el problema no fue resuelto. 
El 5 de marzo de 1972, el gobierno, a través del Decreto 3269, declaró al Instituto como el único comercializador del arrọz, estableciendo un monopolio. Este Decreto causó tanto disgusto entre los molineros como lo habían hecho las leyes agrarias.

Los molineros de arroz empezaron un proceso de cabildeo y presión sobre el gobierno con la intención de cambiar las leyes. Para mantener a los molineros en línea, el Decreto 3919 dio poder al Instituto para perseguir y confiscar embarques de arroz negociados ilegalmente. Sin embargo, los precios se mantuvieron en ascenso y el Instituto tuvo que mantener el programa de importación. Como se muestra en la tabla 6 , los precios de importación para el arroz subieron más del 95 por ciento en 1972-73, y se mantuvieron subiendo hasta el 1974. Esto fue causado por el precio interno que aumentó de 19 a 24 centavos la libra durante este período. ${ }^{7}$ Esto significó una posición muy inestable para el Instituto. No se podía comprar caro en el mercado extranjero y vender barato en el mercado interno. La solución más fácil fue la de ajustar los precios, como un mercado libre hubiese hecho. La tabla 5 muestra un gran aumento en precios, casi 50 por ciento entre 1973 y 1975 . Afortunadamente, los precios internos se mantuvieron al inismo alto nivel. Este punto es un resultado muy diferente del de la proposición del Profesor Schultz. El "mercado político" tiene fuerzas que presionan para mantener los precios al tos y para negar a la masa urbana alimento barato. ${ }^{8}$

Estos elementos proveen un cuadro de un plan de acción muy inestable de la intervención de un mercado importante agrícola de la República Dominicana. Presentaremos las peculiaridades de las "transacciones políticas" hechas en este mercado político.

\section{Mercado Político:}

La historia del Instituto de Estabilización de Precios muestra cómo la hipótesis del Profesor Schultz es parcialmente confirmada como una explicación inteligente de la intervención gubernamental deformada en el mercado del arroz. La historia del Instituto es encuadrada durante la presidencia del Dr. Joaquín Balaguer (1966-78), y podemos explicar las políticas del Instituto como resultado de su filosofía y praxis gubernamentales. Sin embargo, desde 1978, el gobier-

7. Esta crisis está documentada por

Estados Unidos. Agency for International Development. Report on Dominican Republic. W ashington, USAID, 1970

República Dominicana. Secretaría de Estado de Agricultura e Instituto Interamericano de Ciencias Agrícolas. Diagnóstico del Mercado del Arroz en la República Dominicana. Santo Domingo, SEA-1ICA, 1976.

8. Schultz. On Economics... p. 12. 
no del Sr. Antonio Guzmán está a cargo de los asuntos públicos. El ha nombrado a un grupo de funcionarios, técnicamente más preparados, para encargarse de las
instituciones agrícolas.

Pero la existencia de un "mercado político", con los mismo actores y mecanismos del régimen de Balaguer, ha significado una historia continuada de la misma intervención deformante. El modelo de intervención continúa dependiendo de las importaciones para mantener precios estables artificialmente, para lograr el objetivo político de acciones anti-inflacionarias.

Sin embargo, el carácter populista del nuevo gobierno ha llevado a los nuevos burócratas del Instituto a establecer un control más profundo en la producción de arroz, interviniendo en el proceso de molienda a través de la compra de arroz en cáscara y coordinando con los grupos organizados de asentamientos de la Reforma Agraria. Este modelo ha sido modestamente desarrollado.

De cualquier modo, la meta del Instituto es servir a otro sector en el "mercado político", los campesinos. Los mercados políticos pueden ser transformados, porque el actor más poderoso, los molineros de arroz, enfrentaría una nueva organización económica, los molinos cooperativos. El nuevo orden pretende tener una producción agrícola hecha a través de un programa cooperativo de gran escala.

Un "mercado político" tiene funciones de oferta y demanda. En nuestro caso, el gobierno a través del Instituto ofrece una amplia gama de acciones alternativas, desde la no-intervención del poder político-económico y la influencia de los actores políticos que requieran de acciones particulares.

Los elementos de la demanda del mercado político del mercadeo de arroz en la República Dominicana están agrupados en las categorías siguientes:

1. Productores de arroz: Estos están divididos en pequeños -principalmente asentamientos de la Reforma Agraria - y terratenientes. Estos sectores son los defensores más elocuentes del Instituto y están a favor del control de la producción de arroz porque ellos han sido dominados financieramente por los molineros de arroz. La razón principal por la cual éste es el beneficiario preferido de la acción gubernamental es para absorber el desempleo rural y tratar de mantener en línea la migración rural a las ciudades.

2. Molineros de arroz: Este grupo es más un intermediario financiero que un manufacturero, porque gana con especulaciones del inventario. La concentración de grandes corredores en la ciudad de Santo Domingo ha dejado al molino que especule con inventarios. El control financiero y de mercadeo de este grupo lo hizo un blanco fácil de la intervención gubernamental. La acción del gobierno ha 
tratado de mantener a los molineros en el negocio del procesamiento solamente. El Estado ha tratado de asegurar la función financiera a través de un Banco de Desarrollo Agrícola Estatal y la función de mercadeo a través del Instituto.

3. Comerciantes: Este grupo está dividido en mayoristas y detallistas. Este último mantiene una relación de dependencia con el primero por medio de varios planes de financiamiento (crédito a medio y largo plazo). Los mayoristas son los antagonistas más locuaces del Instituto. Ellos favorecen la libre empresa, que les permite importar los artículos necesarios. Los productores se oponen a esto porque les desincentiva la producción. Algunos de los grandes comerciantes son muy importantes en el mercado político porque ellos se beneficiaron del plan de importaciones a través del complejo mecanismo del sistema de cuotas.

4. Consumidores: Este es el grupo más indefenso porque no hay defensores del consumidor en la República Dominicana. Sin embargo, es el tema más mencionado en la prensa y en las intenciones de cualquier ley implementada para intervenir en el mercado.

El Instituto es el foco de todos estos grupos. Los funcionarios son corredores políticos que tratan de maximizar los beneficios políticos para el gobierno aplicando planes de acción diferentes $y$ divergentes para mantener cada grupo "satisfecho", una meta muy evasiva. Las medidas tomadas por los burócratas producirán deformación en la economía que favorece a uno de estos actores económico-político.

Pero los efectos no son los previstos por el Profesor Schultz: el subsidio a los consumidores es sustituido por un objetivo proteccionista de la producción local.

Los precios internos han sido mantenidos a un nivel más elevado que los precios internacionales; en 1976 bajaron un 41 por ciento (ver tabla 6). Las masas urbanas son políticamente menos organizadas que los campesinos rurales. 9 Las presiones rurales son más significativas en las tensiones políticas en la República Dominicana. Esto significa un resultado diferente del de la conclusión de Profesor Schultz.

Las consideraciones políticas del Profesor Schultz están limitadas a la tendencia de las masas urbanas a rebelarse y luchar violentamente de una manera más sistemática que los campesinos. Las presiones rurales, cuando son "sentidas" por los gobiernos a través de ocupaciones de tierras ilegales, revueltas de campesi-

Kenneth E. Sharpe. "Corporate Strategies in the Dominican Republic: The Politics of Peasant Movements". In : James M. Mally, ed. Authoritarism and Corporatism in Latin A merica. Pittsburgh, Pa., University of Pittsburgh Press, 1977. p. 339 375. 
nos y organizaciones, son un elemento significativo en el mercado político. La política gubernamental cambia de acuerdo al caso.

\section{CAPITULO III}

\section{PROPUESTAS PARA EL FUTURQ}

\section{Introducción}

"El Instituto de Estabilización de Precios (apareció) como un eslabón para unir todas las otras instituciones que forman la cadena de actividad primaria en nuestra economía, con meta hacia el productor para garantizarle precios para sus cosechas que le den incentivo a la producción, $y$ al consumidor... para mantener un flujo normal de productos básicos y precios estables accesibles para todos". 10

Esta declaración de los objetivos del Instituto nos lleva a discutir el marco conceptual de las politicas de intervención. Indudablemente, estas dos metas son contradictorias por naturaleza. Para estudiar un nuevo marco conceptual, usaremos un libro escrito hace treinta años por el Profesor Johnson. ${ }^{11}$ Fue escrito con el propósito de discutir las políticas de precios de los Estados Unidos.

\section{Elementos de una Política de Precios}

EI Profesor Johnson presenta dos características de una política económica:

1. Coherencia entre los objetivos de una política, lo que significa que los objetivos deben ser coordinados entre ellos y consistentes con un punto de vista general.

2. Consistencia con las metas del Bienestar Social General, que es la función que permite a los individuos lograr sus propias metas con el menor costo de recursos. ${ }^{12}$ Es evidente que sólo la primera consistencia ha sido tomada en cuen. ta en la declaración interna entre los objetivos del Instituto. Este tipo de consistencia interna entre Jos objetivos diferentes es una preocupación muy limitada.

10. Instituto de Estabilización de Precios. INESPRE : 4 años. Santo Domingo, INESPRE, 1973. p. 2.

11. Johnson, D.G. Forward Prices in Agriculture. Chicago, III, University of Chicago Press, 1947.

12. Idem., p. 16. 
Justifica la crisis explosiva periódica, demandando cambiosen la política por los actores del "mercado político" de! Instituto.

El profesor Johnson enfatiza que sólo la segunda característica es plausible de uso en una política de precio para mercados agrícolas. El mantiene que para lograr los objetivos sociales relacionados con la actividad económica, los resultados deben ser tomados en dos áreas diferentes:

1. La colocación de recursos

2. La distribución de ingresos.

Estas áreas resumen los objetivos de Política Pública, eficiencia y justicia. Los principios generales de la Política de Precios del Profesor Johnson tiene una doble naturaleza, cuatro errores en la formulación de política de precios y cuatro guías para una política de precios saludables. Vamos a considerar estos puntos muy brevemente para establecer una mejor formulación de política para el Instituto. Primero, los cuatro errores:

1. Los precios no deben ser usados como metas logrables. Este error es evidente en las acciones del Instituto. Aun su nombre revela este propósito. La estabilidad de precios es un objetivo político que reduce la eficiencia económica y la producción.

2. La relación de precios no debe ser ligada al pasado. Cada vez que una nueva ley es promulgada estableciendo nuevos precios, ésta es el resultado de una encuesta de precios entre actores. Esta metodología significa la exclusión de nuevas tecnologías y cosechas posiblemente reducidas.

3. Los precios no deben ser usados como elementos de evaluación de la acción administrativa. Este tópico es la prueba más evidente de la práctica errada del Instituto y el Gobierno Dominicano en el período estudiado. La inhabilidad de un administrador del Instituto en conservar los precios tan estables como sea posible significaba un cambio automático del burócrata después que él permitía un aumento de precio.

4. Los precios no deben ser usados como una medida de bienestar en la agricultura. La práctica de establecer los precios sostenidos es reducida a una encuesta de costos de producción previa a la ley definitiva. Ellos usan un promedio del costo más una tarifa arbitraria de beneficios. Esta es una práctica muy peligrosa ya que, la ineficiencia es premiada y la eficiencia castigada. Además, la función empresarial del agricultor es sustituida por un burócrata.

Por otro lado, una política saludable de precios debe ser guiada por cuatro principios: 
1. Los precios deben ser usados como directivas en la economía, el sistema de precios debe ser la fuente de información para permitir a los agricultores usar ne que la intervención y niveles de eficiencia óptimos. Este principio sostiepertimentes a dravés de estas funciones pertimentes al desarrollo agricola, invirtiendo en el capital humano agrícola y produciendo información pertinente sobre las nuevas tecnologías a través de in-
vestigación y de extensión agrícola.

2. La política de precios, como adjunta a la política general monetaria-fiscal, debe ser utilizada para reducir las fluctuaciones en los precios e ingresos agrícolas, la política agrícola es una elemento de política nacional, por lo tanto, otros componentes - políticas fiscal y monetaria-deben ser basados en principios. sólidos, evitando presiones inflacionarias así como comportamiento errático en las inversiones.

3. La política de precios debe ser utilizada para conseguir un considerable grado de estabilidad en el rendimiento de las cosechas individuales y los productos ganaderos; la producción agrícola es, en general, estable. Pero en cosechas particulares tiene fuertes fluctuaciones de precios producidas por fuerzas naturales. La política de precio debe ayudar a los agricultores a soportar estas fluctuaciones.

4. La política de precios debe ser utilizada para reducir la incertidumbre de los agricultores consolidando y transfiriendo la incertidumbre a la economía global, la política agrícola no debe ser aislada del resto de la economía. Así, el riesgo sería minimizado de una manera más eficiente.

Estos cuatro principios son contrarios a la práctica heredada del Instituto. Por esta razón los presentamos como propuestas para el futuro, con la esperanza de que un Instituto más eficiente sea obtenido.

\section{Comentarios Finales}

Hay un interés profundo en este ensayo, y es el de unir el proceso institucional de decisiones con la tasa más amplia de la institucionalización democrática en el caso de la República Dominicana. Es una gran fortuna para el autor encontrar literatura producida en la Universidad de Chicago sobre esta materia.

Esta riqueza de ideas y perspectivas está perdida en este ensayo entre mi suscinto recuento de la historia del Instituto. Sin embargo, el autor prevé un intento de implementar las propuestas dirigidas a reajustar una política de precios en la economía dominicana. 
No hay mejor modo de concluir este trabajo que tomando prestado del Profesor Johnson la observación final de su interesante libro:

Para resumir, los podersos intereses económicos son capaces de hacer mucho para obstruir y desviar los intentos de controlarlos en el interés del resto de la comunidad, y para promover medidas de control que completarán sus propios fines en vez de los de la comunidad. El estado político democrático está particularmente sujeto a estas presiones y obstrucciones, lo que hace aún más difícil una tarea que por su propia naturaleza lo es ya. Si el estado se fortalece contra tales presiones, algo de lo que nosotros estamos acostumbrados a pensar como democracia puede ser perdido. Las burocracias, el poder ejecutivo centralizado y la responsabilidad crecerán. Pero no hay razón para pensar que nosotros tenemos ahora la sustancia del - control popular sobre el gobierno y sus actividades. Las limitaciones principales, ahora como siempre, son esas de la inteligencia y la integridad. ${ }^{13}$

13. J. M. Clark. Social Control of Business. New York, McGraw-Hill, 1939. Como to cita: Johnson, D.G. Forward..., p. 253. 
01. Arvelo-Durán, Tulio. Brazilian Government Policies in Agriculture : The Case of Grain and Soybeans. Chicago, University of Chicago, 1979.

Tesis doctoral.

02. Cramer, Gail L. and Clarence W. Jensen. Agricultural Economics and Agribusiness. New York, Wiley, 1979.

03. Fox, Karl A. y D. Gale Johnson, ed. Readings in the Economics of Agriculture. Homewood, III., Irwin, 1969.

04. Heady, E.O. A Primer on Food, Agriculture and Public Policy. New York, Random, House, 1967.

05. Hopkins, Raymond F. y Donald J. Puchala. The Global Political Economy of Food. Madison, $W$ isconsin, University of $W$ isconsin Press, 1978.

06. Huntington, Samuel P. y Joan M. Nelson. No Easy Choice : Political Participation in Developing Countries. Cambridge, Mass., Harvard University Press, 1976.

07. Instituto de Estabilización de Precios. INESPRE : 4 años. Santo Domingo, INESPRE, 1973.

08. ----. Memoria 1979. Santo Domingo: INESPRE: 1980.

09. International Conference of Agricultural Economists. Decision-Making and Agriculture. Lincoln, Nebraska, University of Nebraska Press, 1977.

10. - - The Future of Agriculture. Oxford, Alden Press, 1974.

11. Lowa State University. Economic Development of Agriculture. Ames, lowa, lowa State University Press, 1965.

12. Johnston, Bruce F. "Agriculture and Structural Transformation in Developing Countries: A Survey of Research". Journal of Economic Literature, 8 (2), jun. 1970.

13. Johnson, D. Gale. Farm Commodity Programs. Washington, D.C., American Enterprise Institute for Public Policy Research, 1973.

14. - - Forward Prices in Agriculture. Chicago III., University of Chicago, 1947.

15. - Policies for Achieving Farm Income Stability. Chicago, III., University of Chicago, 1980

16. Nash, John. Welfare Effects of Price Stabilization : Survey and Modest Extension. Chicago, III., University of Chicago, 1979.

17. Pelletier, William T. Examining Agricultural Policy : A Proposal. Chicago: University of Chicago, 1980. 
18. Schultz, Theodore W. Concept of Enterpreneurship and Agricultural Research. Ames, lowa, lowa State University, 1979.

19. - - "On Economics and Politics of Agriculture". Distortions of Agricultural Incentives. Bloomington, Ind., Indiana University Press, 1979.

20. - - Reckoning the Economic Achievments and Prospects of Low Income Countries. West Lafayette, Ind., Pardue University, 1979.

21. - - - Transforming Traditional Agriculture. New Haven, Yale University Press, 1964.

22. - "The Value of the Ability to Deal with Disequilibria". Journal of Economic Literature, 13: 827-846, sep. 1975.

23. Stanley, Robert C. Food for Peace. New York, Gordon and Breach, 1973.

24. Tool, Marc R. The Discretionary Economy. Santa Mónica, Cal., Goodyear, 1979.

25. Turk, Kenneth L. ed. Some Issues Emerging from Recent Breakthroughs in Food Production. Ithaca, N.Y. Cornell University, 1971.

26. Wamsley, Gary L. y Mayer N. Zald. The Politica! Economy of Public Organizations. Bloomington, Ind., Indiana University Press, 1976.

27. Zald, Mayer N. Organizational Change : the Political Economy of the YMCA. Chicago, III., University of Chicago Press, 1970. 


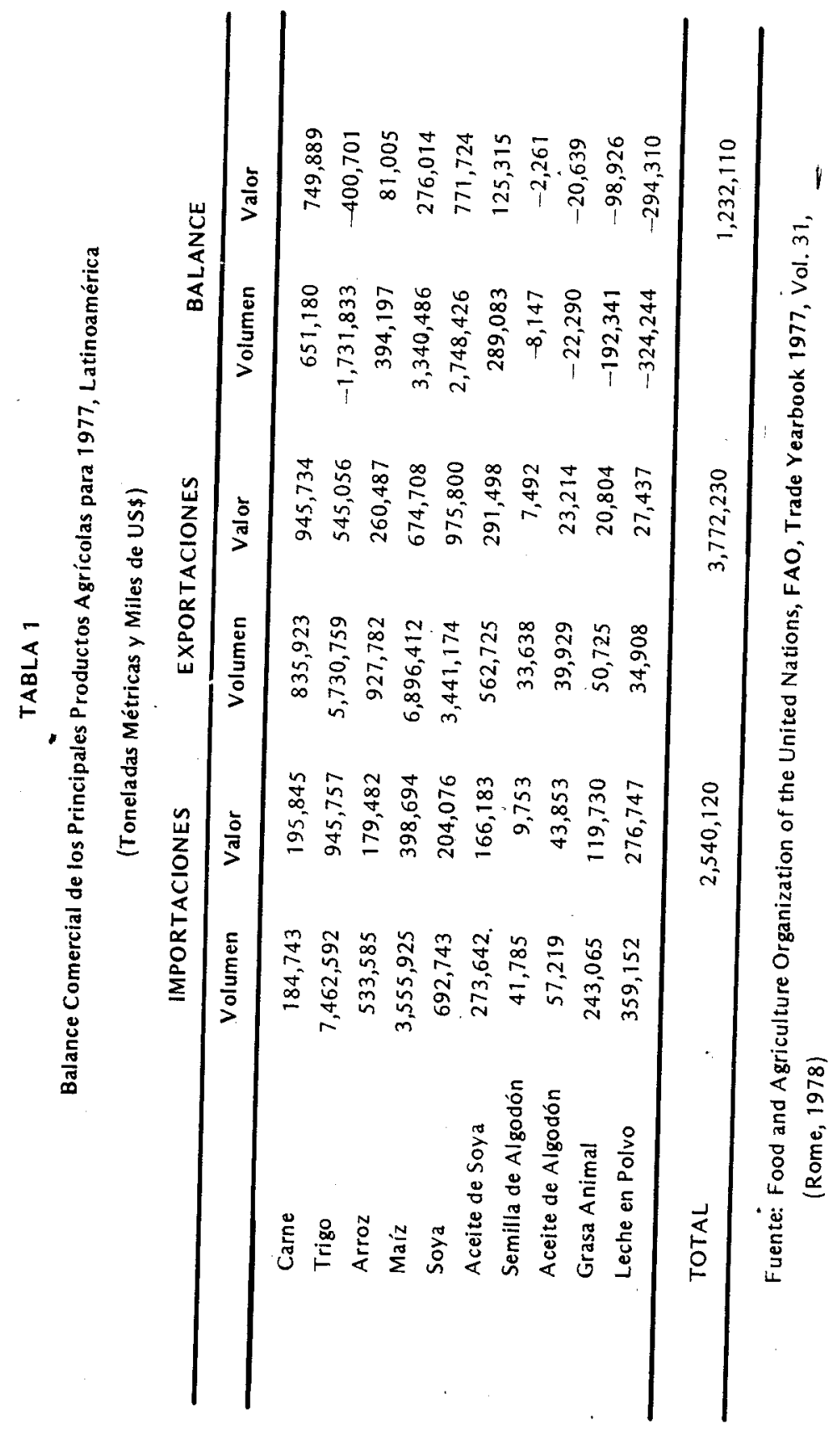


Tabla 2

Comparación entre Precios de Importación y Precios Internos, 1976

(RD/MT)

\begin{tabular}{lccc} 
Producto & $\begin{array}{l}\text { Precio de } \\
\text { Importación (a) }\end{array}$ & $\begin{array}{l}\text { Precio } \\
\text { Interno }\end{array}$ & $\begin{array}{l}\text { Exceso: } \\
\text { Precio Interno/ } \\
\text { Precio Importación (\%) }\end{array}$ \\
\hline Arroz & 427 & 593 (b) & 38.8 \\
Habichuelas & 749 & 515 (c) & -31.2 \\
Maiz & 168 & 186 (c) & 10.7 \\
Acwite Soya & 800 & 1,356 (d) & 69.5 \\
Aceite Mani & 930 & 1,800 (d) & 93.5
\end{tabular}
(a) CIF Santo Domingo
(b) Precio en el Molino
(c) Precio en Finca
(d) Precio al por Mayor

$\operatorname{RD} \$ 1=U S \$ 1$

Tomado de: Banco Mundial, República Dominicana: Problemas del Desarrollo Eco nómico. 
TABLA 3

Importaciones Hechas por el Instituto de Estabilización de Precios

(Miles de TM, Promedio Anual)

\begin{tabular}{|c|c|c|c|c|}
\hline & 1971.73 & 1974.76 & 1977 & $\begin{array}{l}\text { Incremento de la } \\
\text { Producción } \\
\text { Nacional } \\
1971.75\end{array}$ \\
\hline Trigo & 109.3 & 101.9 & n.a. & 0.0 \\
\hline Arroz & 12.8 & 58.7 & 91.0 & 3.0 \\
\hline Habichuelas & 7.5 & 5.7 & 6.1 & 10.3 \\
\hline Maiz & 32.2 & 53.2 & 72.0 & 0.4 \\
\hline Aceite Mani & 13.3 & 6.0 & 18.8 & -6.7 \\
\hline Aceite Soya & 3.7 & 17.0 & 24.0 & n.a. \\
\hline Aceite Algodón & 2.6 & 5.8 & 8.0 & n.a. \\
\hline Total (US\$ mil) & 32.4 & 71.5 & & \\
\hline
\end{tabular}

Tomado de: Banco Mundial, República Dominicana: Problemas del Desarrollo Económico, Cuadro 10, p. 23 
TABLA 4

Producción de Arroz, 1958-78

(1000 Toneladas Métricas)

\begin{tabular}{lcr} 
Año & Cantidad & \%Cambio \\
\hline Promedio 1957-59 & 109 & \\
1960 & 114 & -0.87 \\
1961 & 113 & -1.77 \\
1962 & 111 & 6.31 \\
1963 & 118 & 21.18 \\
1964 & 143 & 14.37 \\
1965 & 167 & 6.58 \\
1966 & 178 & -6.18 \\
1967 & 167 & 8.38 \\
1968 & 181 & 7.73 \\
1969 & 195 & 7.69 \\
1970 & 210 & 0.95 \\
1971 & 212 & 0.94 \\
1972 & 214 & 27.57 \\
1973 & 273 & -5.13 \\
1974 & 259 & -15.44 \\
1975 & 219 & 9.59 \\
1976 & 240 & -22.08 \\
1977 & 187 & 13.37 \\
1978 & 212 & \\
\hline & & \\
\hline
\end{tabular}

Fuente: Unidad de Economía Agropecuaria, Secretaría de Agricultura, Banco Central de la República Dominicana

U.S. Department of Agriculture

Tomado de: Banco Mundial, República Dominicana: Problemas Principales del Desarrollo Económico, Tabia 7.1, p. 378 
TABLA 5

Precio Promedio Mensual del Arroz

(1972-1978)

(Precios corrientes al consumidor)

\begin{tabular}{lllllllll}
\hline & 1972 & 1973 & 1974 & 1975 & 1976 & 1977 & 1978 & 1979 \\
\hline Enero & 0.13 & 0.14 & 0.17 & 0.23 & 0.25 & 0.25 & 0.25 & 0.24 \\
Febrero & 0.14 & 0.14 & 0.17 & 0.23 & 0.24 & 0.25 & 0.25 & \\
Marzo & 0.13 & 0.15 & 0.16 & 0.23 & 0.25 & 0.24 & 0.25 & \\
Abril & 0.14 & 0.17 & 0.16 & 0.23 & 0.24 & 0.24 & 0.25 & \\
Mayo & 0.13 & 0.16 & 0.17 & 0.25 & 0.24 & 0.25 & 0.25 & \\
Junio & 0.14 & 0.16 & 0.19 & 0.26 & 0.24 & 0.24 & 0.25 & \\
Julio & 0.13 & 0.16 & 0.21 & 0.26 & 0.24 & 0.25 & 0.25 & \\
Agosto & 0.14 & 0.16 & 0.22 & 0.25 & 0.25 & 0.25 & 0.25 & \\
Septiembre & 0.15 & 0.16 & 0.22 & 0.24 & 0.25 & 0.25 & 0.25 & \\
Octubre & 0.15 & 0.17 & 0.22 & 0.24 & 0.24 & 0.26 & 0.25 & \\
Noviembre & 0.14 & 0.16 & 0.22 & 0.25 & 0.25 & 0.25 & 0.25 & \\
Diciembre & 0.14 & 0.16 & 0.23 & 0.25 & 0.25 & 0.25 & 0.25 & \\
\hline Promedio Anual & 0.14 & 0.16 & 0.19 & 0.24 & 0.24 & 0.25 & 0.25 & \\
\hline
\end{tabular}

Fuente: Instituto de Estabilización de Precios, Santo Domingo, República Dominicana. 
TABLA 6

Importaciones de Arroz por el Instituto de Estabilización de Precios 1972-1978

\begin{tabular}{lllc} 
Año & $\begin{array}{c}\text { Cantidad } \\
\text { (MT) }\end{array}$ & $\begin{array}{c}\text { Precio Unitario } \\
\text { (RD\$/MT)* }\end{array}$ & $\begin{array}{c}\text { Valor } \\
\text { ('000 RD\$) }\end{array}$ \\
\hline 1972 & 8,851 & 251.3 & $2,224.6$ \\
1973 & 29,686 & 490.1 & $14,551.5$ \\
1974 & 70,301 & 575.4 & $40,452.4$ \\
1975 & 49,510 & 337.9 & $19,165.3$ \\
1976 & 56,245 & 427.0 & $24,015.7$ \\
1977 & 64,474 & & \\
1978 & 10,473 & & \\
& & & \\
\hline
\end{tabular}

Fuente: Instituto de Estabilización de Precios

Tomado de: Banco Mundial, República Dominicana: Problemas del Desarrollo Eco nómico, Tabla 7.6, p. 386 


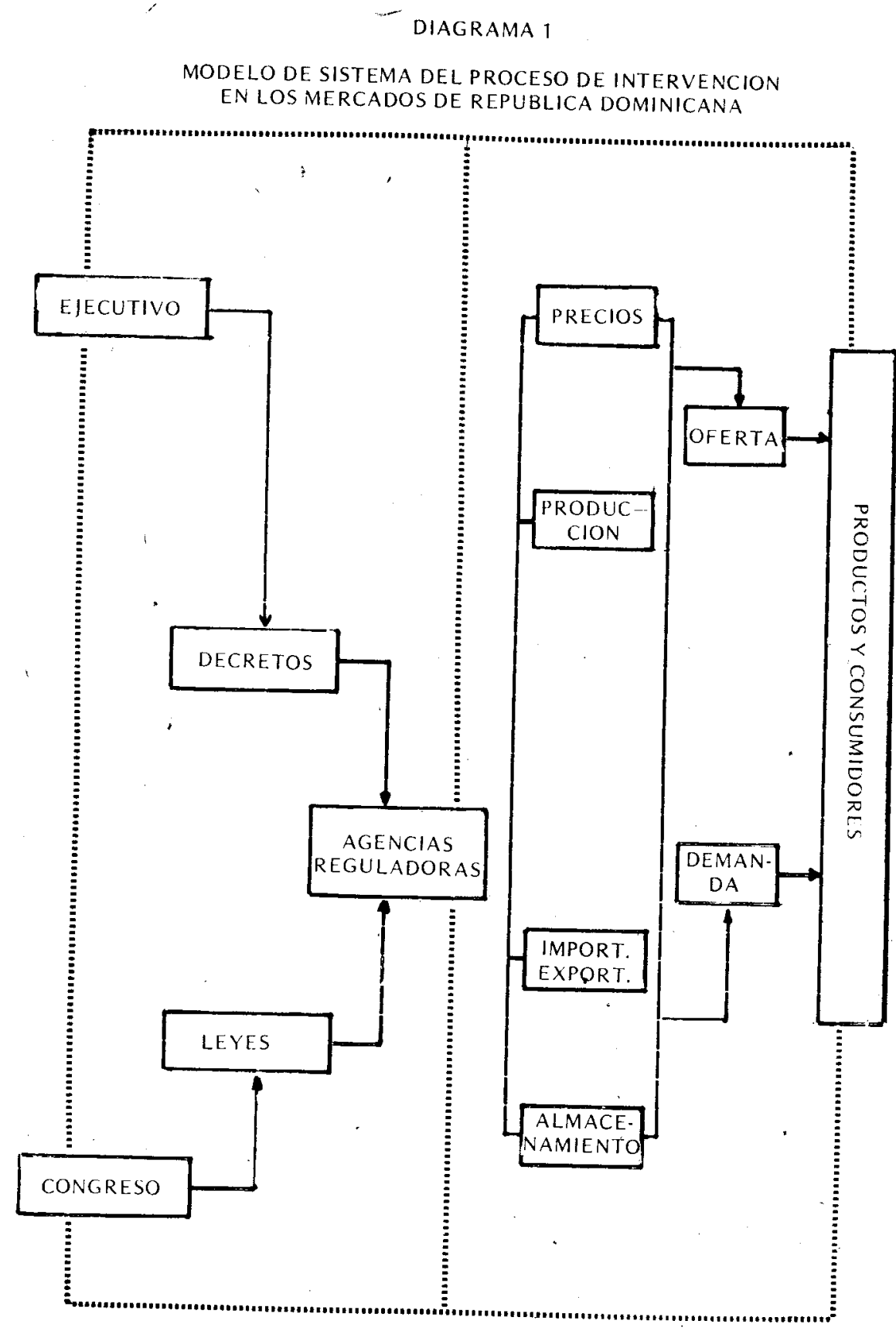




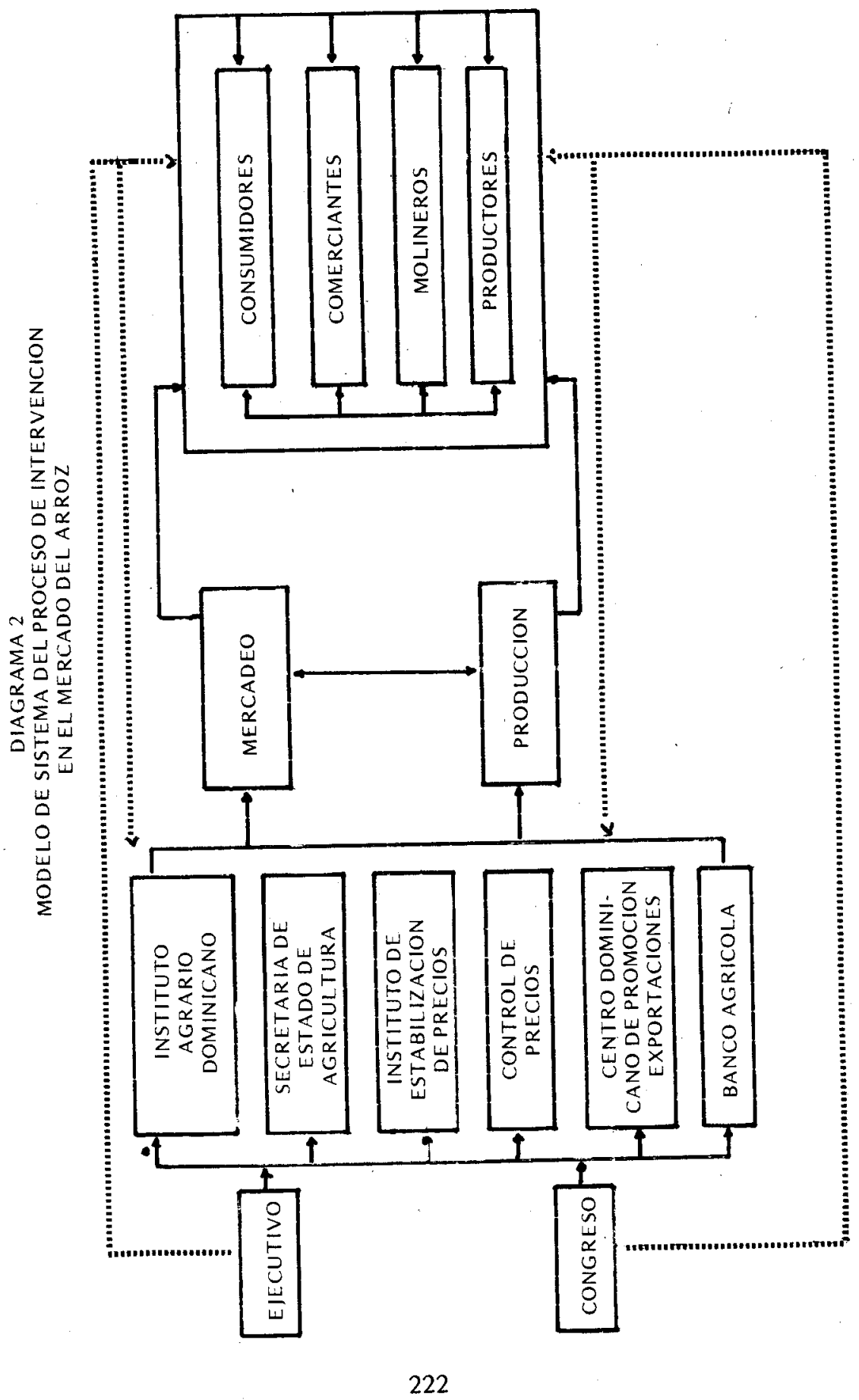

